\title{
Qualitätsmanagement live: Erfahrungen einer Zertifizierung im Bildungsbereich
}

Stefan Wellensiek

(C) Springer-Verlag Wien 2012

Die Errichtung eines Qualitätsmanagementsystems und die Zertifizierung von Unternehmen des Gesundheitswesens sind als Maßnahmen der Qualitätssicherung und Leistungsdarstellung seit Jahren etabliert. Bildungseinrichtungen im Gesundheitswesen und Ausbildungsstätten der Gesundheits- und Krankenpflege sind von dieser Entwicklung jedoch oft ausgenommen. Die positiven Effekte, welche durch ein QM-System und eine Zertifizierung zu erwarten sind, sollten sich auch diese Institutionen zunutze machen, u. a. um ihre Wettbewerbschancen bei qualifizierten Bewerbern zu verbessern und um sich nachhaltig an einem bundesweiten Markt zu positionieren.

Am Beispiel der zentralen Akademie für Berufe im Gesundheitswesen (ZAB), einer Einrichtung der Aus-, Fortund Weiterbildung, wird der Weg der Errichtung eines QMSystems sowie die Zertifizierung nach DIN ISO 9001 und AZAV beschrieben. Im Vortrag werden Erfahrungen des Qualitätsmanagementbeauftragten der Institution als Zeitlinie dargestellt, die von der institutionellen Entscheidung für ein QM-System über den Aufbau des Systems, die Zerti- fizierungen bis hin zu den erfolgreichen Re-Zertifizierungen reicht.

Es werden exemplarisch Probleme benannt, die im Verlauf der Arbeit auf verschiedenen Ebenen zu bewältigen waren. Die Vorteile und der Gewinn, welche sich für die Einrichtung ergeben haben, werden anhand konkreter Beispiele erörtert und aus verschiedenen Perspektiven beleuchtet. Einen weiteren Teil des Vortrags bilden die beispielhafte Betrachtung von Stolpersteinen und Widerständen, welche sich besonders im Bildungsbereich manifestieren. Lösungsorientiert wird der Umgang mit diesen Herausforderungen unter Berücksichtigung der lokalen Bedingungen beschrieben. Abschließend werden die erreichten Verbesserungen den Schwierigkeiten der Einführung und des Alltags gegenüber gestellt, um mit einer qualitativen Bewertung des Qualitätsmanagementsystems für diese Bildungseinrichtung zu enden. Auch hier werden die Sichtweisen der Institution und der Nutzer beleuchtet.

S. Wellensiek $(\triangle)$

Bielefeld, Deutschland 